\title{
Cross-sectional analysis of the associations between fibromyalgia and diabetes mellitus
}

\author{
Adi Lichtenstein ${ }^{1,2}$, Shmuel Tiosano ${ }^{1,2}$, Doron Comaneshter ${ }^{3}$, Howard Amital ${ }^{1,2}$, Arnon D. Cohen ${ }^{3,4}$, \\ Daniela Amital ${ }^{2,5}$
}

${ }^{1}$ Department of Medicine "B” and Zabludowicz Center for Autoimmune Diseases, Sheba Medical Center, Tel-Hashomer, Israel ${ }^{2}$ Tel Aviv University, Sackler School of Medicine, Tel Aviv, Israel

${ }^{3}$ Chief Physician's Office, Clalit Health Services, Tel Aviv, Israel

${ }^{4}$ Faculty of Health Sciences, Siaal Research Center for Family Medicine and Primary Care, Ben Gurion University of the Negev,

Beer Sheva, Israel

${ }^{5}$ Ness-Ziona, Mental Health Center, Beer-Yaakov, Israel

\begin{abstract}
Background: The fibromyalgia syndrome (FMS) is a chronic condition consisting of widespread musculoskeletal pain and tenderness together with mood and cognitive dysfunction. Diabetes mellitus (DM) is a common condition causing significant and detrimental morbidity and mortality. Data on the association between the two conditions is scarce and mainly based on small populations therefore lack solid evidence.

Objectives: To evaluate the association of FMS with DM.

Material and methods: This cross-sectional study used the Clalit Health Services database, the largest Health Maintenance Organization in Israel, serving 4,400,000 members. FMS patients were compared to age- and sex-matched controls regarding chronic comorbid conditions. $\chi^{2}$ and student's t-tests were used for univariate analysis.

Results: The study included 14,296 FMS patients and 71,324 age- and sex-matched controls. The FMS group had a significantly higher proportion of DM patients compared to non-FMS controls (19.8\% and 17.4 respectively; OR $1.17,95 \% \mathrm{Cl}: 1.12-1.23, p<0.001)$.

Conclusions: DM was found to be more common amongst FMS patients compared to matched controls to suggest that the pathophysiology of DM might lead a patient to develop FMS. Consequently, physicians treating DM patients should be aware of the possible risk and asses for clinical signs of FMS in order to diagnose and treat it in time to better their patients' quality of life and disease management.
\end{abstract}

Key words: fibromyalgia, diabetes, pain, neuropathic pain, comorbidity.

\section{Introduction}

Chronic pain is among the most frequent complaints encountered by physicians. The International Association for the Study of Pain (IASP) estimated that chronic pain, including musculoskeletal afflicts about 20\% (10-55\%) of the adult population worldwide [1]. Fibromyalgia syndrome (FMS) is a common form of chronic pain and affecting approximately $2-4 \%$ of the adult female population [2].

FMS is characterized by widespread pain, tenderness and diffuse stiffness. Other associated symptoms include mood, cognitive and functional impairments, such as fatigue, sleep disturbances and headaches [3, 4]. Research indicate that familial, genetic, environmental, endocrine

Address for correspondence:

Howard Amital, Department of Medicine “B” and Zabludowicz Center for Autoimmune Diseases, Sheba Medical Center, Tel-Hashomer, 52621 Israel, e-mail: howard.amital@sheba.health.gov.il

Submitted: 6.10.2018; Accepted: 19.10.2018 
and neurological factors play a role in the emergence of this disorder [2, 5-7].

Several studies have described comorbidities related to FMS [5]. The current report evaluated the association of FMS with diabetes mellitus (DM) using the clinical database of Clalit Health Services (CHS), the largest Health Maintenance Organization (HMO) in Israel. CHS serves a population of about 4,400,000 enrollees. The CHS database allows evaluating "real-life" populations on a large scale, including outpatients and inpatients in primary, secondary and tertiary care centers.

\section{Material and methods}

We conducted a cross-sectional, population-based study using data from CHS. The HMO has a comprehensive, electronic database with continuous, real-time input from pharmaceutical, medical and administrative systems.

In the CHS database, the diagnoses of chronic diseases are based on data derived from hospital and community-based physicians' records. These diagnoses are validated systematically using logistic checks (such as comparing diagnoses from various providers). The validity of the diagnoses in the registry has been shown to be high [8-10].

Patients were defined as having FMS or DM when their medical record contained at least one diagnosis by a physician. All FMS patients in the CHS database were included in our study.

It should be mentioned that in this study more than half of the diagnoses were coded by family physicians (taken from encounters of primary, secondary and tertiary care and often derived from private practice care as well). Thus, a diagnosis coded by family physicians doesn't necessarily mean that a specialist did not perform the actual diagnosis but rather he was not the one to code it into the system.
The control group was randomly selected from CHS enrollees, five control subjects never diagnosed with FMS, who were matched to each FMS patient by gender and age. Data from the CHS database included age, sex, body mass index (BMI), socioeconomic status (SES), smoking status, and diagnoses of chronic diseases, including oncologic disorders.

The distribution of sociodemographic and clinical factors was compared between patients with and without FMS using $\chi^{2}$ test for categorical variables and student's t-test for continuous variables. Crude odds ratios (OR), as well as 95\% confidence intervals $(\mathrm{Cl})$ are presented.

The study was approved by the CHS Ethics Committee, located at Soroka Medical Center, Beer Sheva, Israel.

\section{Results}

The study included 14,296 FMS patients and 71,324 age- and sex-matched controls. FMS patients and controls were at about a mean age of 55 years, and $92.4 \%$ were women (Table I). FMS patients were more likely to be at a low or medium SES and more likely to be smokers than controls were. Their BMI was higher compared to controls (29.1 vs. $28.0 \mathrm{~kg} / \mathrm{m}^{2}$, respectively; $p<0.001$ ) (Table I).

In the control group 12,391 patients (17.4\%) were found to have concomitant DM compared to 2,832 (19.8\%) of the FMS patients. DM was significantly higher in FMS patients with an odds ratio for having concomitant DM at 1.17 (95\% Cl: 1.12-1.23, $p<0.001)$.

\section{Discussion}

This population-based, cross-sectional study was designed to investigate the association between FMS and DM, in a sex- and age-matched analysis.

Table I. Descriptive characteristics of the study population $(n=85,620)$

\begin{tabular}{|lccc|}
\hline Characteristic & $\begin{array}{c}\text { Fibromyalgia } \\
n=14,296\end{array}$ & $\begin{array}{c}\text { No fibromyalgia } \\
n=71,324\end{array}$ & $\begin{array}{c}\text { Odds ratio } \\
(\mathrm{Cl})\end{array}$ \\
\hline Age & $55.2 \pm 13.9$ & $56.0 \pm 13.7$ & $1.00(0.99-1.00)$ \\
\hline Females & $13210(92.4 \%)$ & $65910(92.4 \%)$ & $1.00(0.93-1.07)$ \\
\hline BMI & $29.1 \pm 6.20$ & $28.0 \pm 6.01$ & $1.03(1.03-1.03)$ \\
\hline Smoking & $4679(32.7 \%)$ & $19987(28.0 \%)$ & $1.25(1.20-1.30)$ \\
\hline SES & & & 0.978 \\
\hline Low & $6669(46.8 \%)$ & $27747(39.2 \%)$ & 0.001 \\
\hline Medium & $5397(37.9 \%)$ & $27499(38.8 \%)$ & $0.82(0.78-0.85)$ \\
\hline High & $2182(15.3 \%)$ & $15554(22.0 \%)$ & $0.58(0.55-0.61)$ \\
\hline Diabetes & $12391(17.4 \%)$ & $2832(19.8 \%)$ & $1.17(1.12-1.23)$ \\
\hline
\end{tabular}

BMI - body mass index $\left(\mathrm{kg} / \mathrm{m}^{2}\right)$; SES - socioeconomic status 
According to the $\mathrm{WHO}$, in 2014, 422 million people in the world had diabetes - a prevalence of $8.5 \%$ among the adult population. The prevalence of diabetes has been increasing over the past 30 years as well as the associated risk factors such as being overweight or obese [11].

A publication by the Israel Center for Disease Control (ICDC) estimates that DM is today is the fourth leading cause of death in Israel. In 2013, 5\% of deaths in men and $5.9 \%$ in women were attributed to diabetes and its' complications. According to the Israeli National Health Survey (INHIS-3) DM type 2 affects $8.8 \%$ of men and $8.1 \%$ of women over the age of 21 years escalating to rates of $27.3 \%$ in men and $20.5 \%$ in women older than 65 [12]. Beyond the age of 55 the rates in men exceeds those reported in women.

DM is associated with many complications that eventually result in high rates of morbidity and mortality including hypoglycemia episodes, diabetic coma, neuropathy, nephropathy, hypertension, stroke, hyperosmolar hyperglycemic nonketotic syndrome, diabetic ketoacidosis and ketones, amputation, gastroparesis, microangiopathy, macrovascular disease and many others [11]. It is highly likely that some of the complications mentioned above may cause symptoms of pain, mood and sleeping disturbances.

Most of the literature to date shows higher rates of FMS in patients with DM ranging from $17 \%$ to $23.3 \%$ [13-16]. Results from our study correspond with the literature as DM was found in a proportion of $19.4 \%$ of patients with FMS with an OR of 1.17 compared to controls.

Results from Tishler et al. [14] on 100 consecutive, unselected DM patients (45 DM type 1 and 55 DM type 2) and 50 randomly recruited healthy hospital staff as controls, revealed an association of FMS with elevated $\mathrm{HbA}_{1 \mathrm{c}}$ levels as well as a correlation between $\mathrm{HbA}_{1 \mathrm{c}}$ levels and the number of tender points. DM patients with FMS have more tender points and more complaints of widespread pain compared with controls [13, 17, 18]. Italian researchers even suggested that insulin resistance might be a risk factor for memory deficit among patients with FMS through sympathetic nervous system activation and hypothalamic pituitary axis dysregulation [19].

FMS and diabetic peripheral neuropathy share many aspects. A study of 100 patients with type 2 DM patients showed that peripheral neuropathy was detected in $61.9 \%$ of patients with DM and FMS compared to only $2.5 \%$ in patients with DM without FMS [15].

The main limitation of this study was the fact that we relied on diagnoses appearing in the patients' medical records. By nature, we had no confirmation of their validity according to the classification criteria applied in clinical studies. The CHS database was originally created to accommodate data for clinical proposes, therefore, it was designed to act as a data registry according to diagnostic criteria of disorders but rather to code diseases according to the International Classification of Diseases (ICD) for statistical, economical and administrative purposes. However, the validity of the diagnoses as exhibited in previous studies have been shown to be high and reliable [8, 9, 20-24].

The main strength of this study is its large sample size. The CHS database that we used in this study covers more than half of the population in Israel. Thus the data represent the entire general population composed by the matrix of the diverse ethnicities, socioeconomic backgrounds and geographical differences.

\section{Conclusions}

In conclusion, both DM and FMS are common in the general population. Our study indicates that FMS patients seem to be prone to have concomitant DM regardless of which condition was discovered first. Consequently, physicians treating DM patients should be aware of the symptomatology of FMS, it's association with obesity and refer selected patients to a rheumatologist to properly diagnose and treat FMS.

Prof. Arnon Cohen received research grants from Janssen, Novartis, AbbVie, Janssen and Sanofi. Arnon Cohen served as a consultant, advisor or speaker to AbbVie, Amgen, Boehringer Ingelheim, Dexcel Pharma, Janssen, Kamedis, Lilly, Neopharm, Novartis, Perrigo, Pfizer, Rafa, Samsung Bioepis, Sanofi, Sirbal, and Taro. Prof. Howard Amital received research grants from Janssen, AbbVie, Pfizer, Gilead as a consultant, advisororspeaker to AbbVie, Boehringer Ingelheim, Janssen, Neopharm, Novartis, Perrigo, Pfizer, Sanofi, and Taro.

The rest of the authors declare no conflict of interest.

\section{References}

1. Queiroz LP. Worldwide Epidemiology of Fibromyalgia. Curr Pain Headache Rep 2013; 17: 356.

2. Grodman I, Buskila D, Arnson Y, et al. Understanding fibromyalgia and its resultant disability. Isr Med Assoc J IMAJ 2011;13: 769-772.

3. Jones GT, Atzeni F, Beasley M, et al. The prevalence of fibromyalgia in the general population: a comparison of the American College of Rheumatology 1990, 2010, and modified 2010 classification criteria. Arthritis Rheumatol 2015; 67: 568-575.

4. Wolfe F, Clauw DJ, Fitzcharles M-A, et al. The American College of Rheumatology preliminary diagnostic criteria for fibromyalgia and measurement of symptom severity. Arthritis Care Res 2010; 62: 600-610. 
5. Lichtenstein A, Tiosano S, Amital H. The complexities of fibromyalgia and its comorbidities. Curr Opin Rheumatol 2018; 30: 94-100.

6. Amital D, Fostick L, Polliack ML, et al. Posttraumatic stress disorder, tenderness, and fibromyalgia syndrome: are they different entities? J Psychosom Res 2006; 61: 663-669.

7. Yavne Y, Amital D, Watad A, et al. A systematic review of precipitating physical and psychological traumatic events in the development of fibromyalgia. Semin Arthritis Rheum 2018; 48: 121-133.

8. Yavne $Y$, Tiosano S, Watad A, et al. Investigating the link between ischemic heart disease and Behcet's disease: A cross-sectional analysis. Int J Cardiol 2017; 241: 41-45.

9. Dagan A, Segal G, Tiosano S, et al. Coexistent malignant conditions in rheumatoid arthritis - A population-based cross-sectional study. Int J Clin Pract 2017; 71: e12929.

10. Merdler-Rabinowicz R, Tiosano S, Comaneshter D, et al. Comorbidity of gout and rheumatoid arthritis in a large population database. Clin Rheumatol 2017; 36: 657-660.

11. World Health Organization. Global report on diabetes, 2016. Available from: http://apps.who.int/iris/bitstream/handle/10665/204871/9789241565257_eng.pdf;jsessionid=CB4F91AF9F1063F5B50E7D42B61F6DCB? sequence $=1$

12. Israel Center for Disease Control. Highlights of health in Israel, 2017. Available from: https://www.health.gov.il/publicationsfiles/highlights_of_health_in_israel2016.pdf

13. Wolak T, Weitzman S, Harman-Boehm I, et al. Prevalence of fibromyalgia in type 2 diabetes mellitus. Harefuah 2001; 140 1006-1009, 1119, 1120.

14. Tishler M, Smorodin T, Vazina-Amit M, et al. Fibromyalgia in diabetes mellitus. Rheumatol Int 2003; 23: 171-173.
15. Mirghani HO, Elbadawi AS. Are diabetes mellitus, restless syndrome, and fibromyalgia related? Indian J Basic Appl Med Res 2016; 5: 208-215.

16. Tugrul G, Coskun NC, Sarpel T, et al. THU0559 Frequency of fibromyalgia syndrome in patients with type 2 diabetes mellitus. Ann Rheum Dis 2016; 75 (Suppl 2): 394-394.

17. Yanmaz MN, Mert M, Korkmaz M. The prevalence of fibromyalgia syndrome in a group of patients with diabetes mellitus. Rheumatol Int 2012; 32: 871-874.

18. Patucchi E, Fatati G, Puxeddu A, Coaccioli S. Prevalence of fibromyalgia in diabetes mellitus and obesity. Recenti Prog Med 2003; 94: 163-165.

19. Fava A, Plastino M, Cristiano D, et al. Insulin resistance possible risk factor for cognitive impairment in fibromialgic patients. Metab Brain Dis 2013; 28: 619-627.

20. Watad A, Bragazzi NL, Tiosano S, et al. Alzheimer's Disease in Systemic Sclerosis Patients: A Nationwide Population-Based Cohort Study. J Alzheimers Dis 2018; 65: 117-124.

21. Watad A, Bragazzi NL, Adawi M, et al. Anxiety disorder among rheumatoid arthritis patients: Insights from real-life data. J Affect Disord 2017; 213: 30-34.

22. Sharif K, Watad A, Tiosano S, et al. The link between COPD and ankylosing spondylitis: A population based study. Eur J Intern Med 2018; 53: 62-65.

23. Yavne $Y$, Tiosano S, Ben-Ami D, et al. Giant cell arteritis and inflammatory bowel disease - Is there a connection? Results from a population-based study. Autoimmun Rev 2018; 7: 1134-1137.

24. Tiosano S, Yavne Y, Gendelman O, et al. Stroke among rheumatoid arthritis patients: Does age matter? A real-life study. Neuroepidemiology 2017; 49: 99-105. 